\title{
EVALUATION OF GREEK PUBLIC HOSPITAL WEBSITES
}

\author{
Fotini Patsioura, Spyros Kitsiou and Angelos Markos \\ Department of Applied Informatics, University of Macedonia, 156 Egnatia Str., Thessaloniki, Greece \\ \{fotpat,skitsios,amarkos\}@uom.gr
}

Keywords: e-Health, e-Government, Evaluation, Criteria, Hospital, Websites.

Abstract: Hospital web sites need to operate as effective web resources of information and interactive communication mediums to address the multifaceted requirements of their main stakeholders, (i.e. patients, healthy citizens, healthcare professionals and local communities). In case of public hospitals, web sites should also be considered as a mean to provide governmental electronic services. The main objective of this study is to provide an evaluation framework for hospital web sites. The proposed framework focuses on three main criteria: information, communication and electronic services. A total of 53 Greek hospital web sites within the Public Sector were evaluated based on a 67-item scale. The main findings of the study were the following: (a) the majority of the web sites in the analysis failed to support necessary web-based transactions between the institutions and their stakeholders (b) web sites are lacking important features regarding provision of information and communication practices. Improvements on interaction and information quality are necessary in order for the institutions to provide a viable and useful online environment for patients and citizens.

\section{INTRODUCTION}

Direct healthcare providers need to deliver satisfactory services in a specialized field which involves a great number of stakeholders with different concerns, needs and requirements. So far, hospitals' policies have been focused on providing health and medical services to the public. Limited attention has been given on their responsibility to provide useful, accurate health information of high quality to their key publics mainly by facilitating interactive communication with patients, citizens, physicians and community services.

To date, hospitals are turning increasingly towards the Internet and develop their own web presence in order to enhance the provision of information and also employ interpersonal and interactive communication practices. Moreover, public and private health institutions acknowledge the importance of building relationships with current or potential patients by providing a friendly and functional online environment. In this setting, public hospitals should go one step forward taking into account their authority in providing and supporting governmental or public electronic services such as provision of documentation, facilitation of routine transactions (e.g. appointment scheduling, billing procedure handling) and gathering of valuable feedback in order to evaluate the quality of their overall services. Internet-based consumer services provided through hospital web sites are necessary to improve relationship management, citizens' convenience and patient care, but also required in order to enhance the operation of these web sites as a complete public marketing instrument.

Many evaluation instruments on hospital and public sector web sites were examined and analysed. Most of them focus on information content and communication aspects. Little research has been conducted regarding their capability to provide interactive and transactional services. This study aims at providing an evaluation framework for hospital web sites that examines their overall performance as reliable information web resources and advanced interactive and transactional e-service mediums. It should be mentioned that the proposed framework mainly focuses on the capabilities of hospital web sites to meet the requirements and needs mainly of their key stakeholders (i.e. patients and citizens). It is in the authors' opinion that this approach should provide better understanding and analysis of the hospital web sites' multidimensional role. 


\section{LITERATURE REVIEW}

Mukherjee \& McGinnis (2007) discussed how important is for healthcare providers to utilize the internet and the new technologies in order to improve healthcare services, further implement their facilities, and also support two main activities of their stakeholders on the Internet, information searches and communication requests. For hospitals, web sites are the key platform to accomplish information provision and communication services outside their facilities. Research has shown that people searching for heath care information are becoming more demanding and sophisticated with regards to information and web site quality (Bodkin and Miaoulis, 2007). Although hospitals have paid attention to this trend, limited action has been undertaken to take advantage of the Internet capabilities in order to meet their stakeholders' requirements and needs and deliver technologically useful services.

The analysis of current situation by evaluating private and public hospitals' web sites is essential in order to identify the gaps and improve their overall performance. Most research on this field is focused on information quality and usability issues. Norum (2001) evaluated cancer related hospital web sites based on information provision (general and contact information) and web sites accessibility aspects (browser presence and English version web site). Randeree and Rao (2004) provided an evaluation instrument for American hospital web sites using eight key criteria factors: access, audience, accuracy, timeliness, content, authority and privacy to assess both the presentation and transaction of information, but also the functionality of the web sites. Results derived from this study revealed significant gaps in terms of information accuracy, currency and customisation. Hospital web sites' content remains a major issue, especially when consumers of healthcare information on the internet remain sceptical towards the source of information (Huntington et al, 2004).

Nevertheless, hospital web sites should fulfil objectives beyond the delivery of accurate and trustworthy information. Gruca \& Wakefield (2004) emphasize on the importance of communication processes in facilitating information transactions between institutions and patients or other stakeholders. Provision of multiple communication channels such as electronic mail, online forms, and discussion forums are necessary to establish direct personal interaction and satisfy current or potential patients who expect an easy way to contact their physicians or other facilities within the hospital. According to Smith (1997) a friendly interaction environment for users of hospitals' web sites should be considered as an essential online service by the providers. For hospitals the utilisation of the Internet is the only money-saving way to "bring patients and services and clinicians closer together" and most significantly to build patient relationships in many ways that have not been possible before (Lewers, 2003). While building relationships is an essential goal for corporate and commercial web sites (Keating et al, 2003; Boisvert \& Caron, 2006), in case of hospitals' web sites it remains an underestimated element. So far, limited academic research was conducted to determine the quality of communication processes delivered to health consumers on the Internet by hospitals' web sites.

In terms of web site evaluation, it is apparent that previous research regarding hospitals' web sites is affected by the commercial web sites' evaluation research. Criteria like design, accessibility, readability, interactivity, navigation and content have been widely used in the past for health related and hospitals' web sites (Smith, 1997; Liddell, 2005; Randeree \& Rao, 2004; Llinas et al, 2007). Although useful, this approach could be inadequate to produce complete measures as it fails to take under consideration the electronic services aspect. In the future, more and more hospitals will invest on electronic services through their web site to support functions and activities such as information search, appointment scheduling, problem solving policies, billing and payment procedures. For private hospitals this action is necessary in order to enhance their consumers' satisfaction, while for public hospitals is mandatory as part of their authentication towards standards approval procedures (Ancarani, 2005). In both cases, electronic services are the next step to enhance web sites' performance while engendering health care consumers' support.

\section{EVALUATION FRAMEWORK}

The main objective was to develop a conceptual framework that can be used in the evaluation of the overall performance of Hospitals' web site towards the multiple objectives of their establishment.

Hospitals websites should ultimately provide healthcare consumers with the ability to perform the following main activities: gather accurate and reliable information for decision-making purposes, contact and interact either online or directly with the hospital's medical and administrative personnel, and 
last but not least, perform a range of online transactional and interactive activities.

Based on that, the proposed framework identifies two key categories for hospitals' web site evaluation based on healthcare consumers' needs and requirements, defined in table 1 :

- Information

- Communication and Transaction

Table1: Criteria categories.

\begin{tabular}{|c|l|}
\hline $\begin{array}{l}\text { Criterion } \\
\text { Category }\end{array}$ & \multicolumn{1}{|c|}{ Definition } \\
\hline \multicolumn{1}{|c|}{ Information } & $\begin{array}{l}\text { Web site's capability to provide } \\
\text { adequate information about the hospital } \\
\text { and general health }\end{array}$ \\
\hline $\begin{array}{l}\text { Communication } \\
\text { and Transaction }\end{array}$ & $\begin{array}{l}\text { Web site's capability to facilitate direct } \\
\text { communication among the hospital and } \\
\text { health consumers and enable interactive } \\
\text { transactions to enhance consumer } \\
\text { services }\end{array}$ \\
\hline
\end{tabular}

Information. According to Jadad et al (2001), in the new age of e-healthcare, academic, professional and governmental health organisations need to communicate health information from institutions to patients' home. In case of hospital web sites, the provision of information has a dual purpose. Firstly, to enable decision-making toward the selection of the most appropriate provider and specific medical services. Secondly, to serve as a reliable source of either general or specific health information, in order to improve health and assist consumers in the management of their own health. Previous research addressed the issue of citizens' and consumers' trust on healthcare information (Huntington et al, 2004).

Patients should be free of concerns and insecurities when gathering information about the hospital or general healthcare information. In health consumers' mind, trustworthy healthcare information comes when visiting a recognised institution (Lin \& Umoh, 2001). A recent study, conducted by the Health On the Net Foundation (Health On the Net Foundation, 2004), revealed that approximately $75 \%$ of American and European patients and healthcare professionals prefer to search and obtain information from hospital-sponsored websites.

However, it is equally important and should be in web site developers' concern to facilitate the gathering and selection of accurate and validated health information by the citizens in terms of usability issues. Therefore, the proposed framework, regarding the information content focuses on the following main criteria groups: (1) hospital generic information (2) hospital specific information (3) health information, (3) currency of information, (4) disclosure of credentials (for the organisation, and (5) links to external resources.

Communication and Transaction. A virtual environment is effective in terms of communication when online consumers' or citizens feel the familiarity and personal attention of face-to-face communication. Especially, for hospitals' web sites communication between current or future patients and physicians will strengthen the hospital's relationship marketing policy. The Internet facilitates interactive transactions and interpersonal communication with the use of a variety of technologies and practices. The provision of multiple communication channels is a means for accomplishing direct personal interaction with limited effort and time. When evaluating a hospital's web site, it is vital to examine its capabilities to respond to consumers' inquiries and allow their direct contact with the hospital's medical, managerial or administrative services. The proposed framework investigates the provision of adequate contact and communication information and the site's capability to facilitate direct, interactive and personal communication.

Regarding the aspect of transactions, in commercial web sites online transactions services are provided to support online customers throughout their overall shopping experience (Zeithaml et al, 2002). In the public sector, online transaction services encompass supportive activities, including provision of information and documentation, complaints management, and sometimes more specific transactions such as supplies handling, contracting and billing (Ancarani, 2005). With regards to hospitals, Gruca and Wakefield (2002), along with others (Llinas et al., 2008; Gallant et al., 2007; Zingmond et al., 2001) describe online transactions as the capabilities of hospital websites to facilitate interactive communication between the institutions and their stakeholders (e.g. via online forms, online forums, support groups, chat boards, etc.) or more sophisticated such as online appointment scheduling, online reporting of clinical test results, online payments and management requests. Hospitals should go one step forward taking advantage of the unique characteristics of the Internet and the information and communication technologies in general with the aim to deliver services electronically to hospitals' main stakeholders. Facilitation of information search and selection, web site personalization, the delivery of 
documents electronically, fully online transactions such as billing procedures and payment processes are some of the facilitating and supportive services that should be the evolution of a transactional and interactive online hospital website environment.

Therefore, the proposed framework, regarding the aspects of communication and transactions focuses on the following main criteria groups: (1) contact and communication content, (2) interaction and transaction mechanisms, (3) user support and ease of use.

Based on the above discussion, Figure 1 illustrates the proposed evaluation framework. Each of the two aforementioned criteria categories that comprise the proposed evaluation framework are further analysed in 9 criteria groups. These 9 groups encompass a range of web site features to support the evaluation of hospital websites.

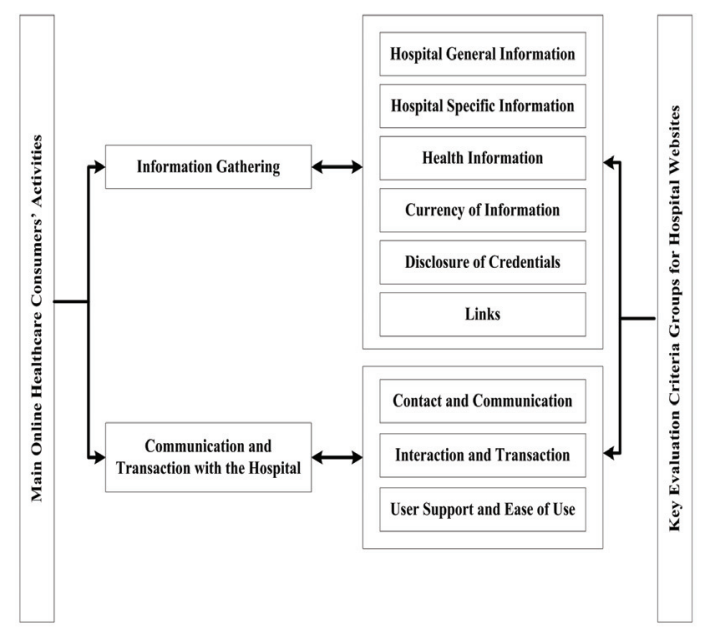

Figure 1: Evaluation Framework.

\section{METHODS}

Between June and July of 2008, a thorough evaluation study to assess the information and transactional features of Greek Hospital Web Sites was carried out.

For the identification of public hospital websites,

we used the online national directory of the Greek Health Ministry. When no hospital website was identified, a telephone call was made to the hospital to confirm that there was no website.

Based on the 9 criteria groups of the evaluation framework, an instrument comprising of 67 explicit evaluation criteria were developed and utilized for the assessment of the websites. The websites were evaluated based on the presence (1) or absence (2) of each criterion. This approach was chosen by the authors to minimize subjectivity by the evaluators during the assessment process. The two authors of this paper (F.P. and S.K.) carefully examined the websites using a standard browser (Internet Explorer, Microsoft) and completed the evaluation instrument during their in-depth visit in order to provide reliable results.

For the final database and the recording of the results the authors used Microsoft Excel 2003 and the SPSS version 15 for statistical analysis.

Next, to assess the reliability of the instrument, coefficients of internal consistency were used. Precisely, internal consistency was tested by calculating Cronbach's alpha for the dichotomous items of the information and the interactiontransactional criteria groups. Table 2 presents the reliability results, which generally establish the correctness of the proposed measurement structure. The Cronbach's alpha values for the two criteria groups that were used in the study are very satisfactory and generally establish the correctness of the instrument structure and thus the structure of the proposed evaluation framework.

Table 2: Reliability Results (Cronbach's alpha).

\begin{tabular}{l|c|}
\hline \multicolumn{1}{|c|}{ Criteria Categories } & Cronbach alpha \\
\hline $\begin{array}{l}\text { Information criteria groups (6) } \\
*\end{array}$ & $0.804(41) * *$ \\
\hline $\begin{array}{l}\text { Interaction and Transaction } \\
\text { criteria groups (3) }\end{array}$ & $0.701(26) * *$ \\
$*$ *Number of criteria groups \\
$* *$ Number of items/criteria
\end{tabular}

\section{RESULTS}

Out of the 131 public hospitals that operate in the context of the Greek public healthcare system, a total of 57 hospital web sites $(43,5 \%)$ were identified. Of the 57 public hospitals, 39 were general hospitals $(68,4 \%), 6(10,5 \%)$ were operating both as primary care health centres and general hospitals, 5 were teaching hospitals $(8,8 \%)$, and 7 $(12,3 \%)$ were specialty hospitals. Regarding their size in terms of clinical beds, 10 hospitals $(17,5 \%)$ were small in size (25-100 beds), 13 (22,8\%) were small to medium size hospitals ranging from 101250 beds, $29(50,9 \%)$ hospitals were medium to large (251-750 beds), and 5 hospitals were large $(8,8 \%)$ having more than 750 clinical beds.

Information. On the first criteria group of the information category, which evaluated the presence 
or absence of "general hospital information", the vast majority of the websites consistently reported information about the history (93\%), the location $(77 \%)$, and the size $(65 \%)$ of the organization in terms of number of beds. Also, $65 \%$ of the websites provided information about the structure of the organization by illustrating their organizational chart. Many hospitals take advantage of the web to apprise visitors with current news and announcements (65\%) about the institution. On the other hand, it was found that most hospital websites fail to guide visitors on how to get to the hospital by making available information on transportation means $(28 \%)$ and lodging or travelling, including maps and directions (35\%). For large hospitals in large cities that often have many locations, numerous buildings and, perhaps multiple entrances for the same buildings this was considered to be an important limitation.

Regarding the second group, which encompasses criteria on specific hospital information that play an important role in the decision making process of health consumers towards the selection of the most appropriate provider, it was found that specific information regarding the range and type of clinical and administrative departments and services were present at the majority of the web sites, $92 \%$ and $74 \%$ of the times respectively. However, basic information regarding the clinical personnel of the hospital (e.g. physicians and nurses) such as directory lists with names and specialties were present less than half of the times. Moreover, important information about physicians' background and experience was significantly limited $(2 \%)$. Information about hospitalization guidelines such as admission procedures $(37 \%)$, necessary documents $(40 \%)$ and information about insurance coverage or plans (28\%) were also found at low frequencies. These results are considered to be very disappointing. According to Clines and Haynes (2001), information about core competencies of physicians, hospital specialties, insurance coverage, and general hospitalization guidelines can influence consumers, in terms of decision making and selection of the most appropriate healthcare provider based on their situation. Also, a recent study undertaken by Gallant et al (2007) found that access to physicians' information, reputation, and background experience can encourage the creation of trust, which is considered to be a vital aspect of a hospital's relationship with its public (Hesse et al., 2005). Therefore, the aforementioned results demonstrate that Greek hospital websites fail to create basic elements of trust with their current and potential customers.

Health information access is also considered to be an important service that hospital web sites can provide to their uses. Unfortunately, the vast majority of the Greek hospital websites that were surveyed did not provide any kind of either general or specific health information. Only $28 \%$ had a devoted section for consumer health information regarding specific diseases, research studies or consumer-oriented educational material.

Concerning currency of information, less than a third $(29 \%)$ of the websites in the sample provide dates of last page updates and only $16 \%$ of them provide dates of page creation. This is an important drawback taking into account that visitors need to be certain at all times about the currency of specific information such as visiting hours, hospitalization costs, health information and duty hours of hospitals.

As regards to disclosure of credentials, unfortunately only $5 \%$ of the evaluated websites include information on credentials or certifications of services about the institution (e.g. markers of excellence, awards, quality credentials, ISO, EFQM, etc.) Such low levels demonstrate that the majority of Greek healthcare organisations do not fully comprehend the vital significance of credibility appraisal toward the development of consumers' trust. Moreover, it is also a negative observation that only $35 \%$ of the websites provide information about the participation of the institution in scientific studies and its research contribution which could enhance the institution's image on the community.

Links to other health care websites or general health information sites were provided only by less than half of the surveyed hospitals (37\%), while the frequency of links to other healthcare organizations was also low (32\%). However, having external links to health and medical information is considered to be an important feature that hospital websites should have, in order to strengthen their usefulness.

Communication \& Transaction. As for contact and communication content, findings reveal that most of the websites provide adequate communication information to facilitate direct and interpersonal contact with the institution's faculty but mainly about traditional communication channels (telephone, fax). Specifically, almost all websites $(93 \%)$ provide a general phone number and $63 \%$ of them include a fax number. In addition, telephone numbers of administration employees $(68 \%)$ and physicians $(65 \%)$ are often included. However, communication via e-email is not 
supported by the majority of the websites. Almost half of the websites $(45 \%)$ do not include a central email address. Additionally, physicians e-mail addresses are included only on $19 \%$ of the websites and employees' email addresses are provided by $38 \%$. Finally, it is worth mentioning that regarding scheduling appointments, $72 \%$ of the websites in the sample include the phone numbers of outpatient clinics.

Interaction and Transaction mechanisms were unfortunately scarce or absent in the vast majority of the hospital websites. Specifically, it was found that only $37 \%$ provided a central online communication form to address consumers' inquiries about hospital services, while near $10 \%$ included online forms to enable interpersonal communication with physicians (9\%) and administration employees (12\%). Moreover, there was almost a complete absence of personalization features $(5 \%)$ that could enable the provision of targeted information, content or services to the website users. Personalization features can encourage the commitment of the audience toward the hospital website and the organization itself, while also according to Gallant et al (2007) "providing a personalized website for indivinduals, upon either logging into or automatic recognition of user arrival, can increase the usefulness of a website for its audience". Only 5\% of the websites incorporate real-time interaction mechanisms such as discussion forums or chatboards to enable direct online communication and exchange of views between the hospital and the consumers or even among consumers. Also, none of the websites in the sample provide any kind of advanced transaction mechanisms for online appointment scheduling, settling of financial accounts (e.g. online service payments), and online access of medical test results.

Regarding "user support and ease of use" criteria which examine the presence of basic functionality and usability features and tools, results show the lack of significant mechanisms that support ease of navigation and access. Specifically, only 3\% of the websites include a "help" page, and only $5 \%$ provide information on browser compatibility. Almost a third of the web sites provide a search engine to facilitate information gathering and less than 5\% support advance search engine features. Furthermore, less that half (38\%) include a site map on their webpage. With respect to accessibility, only $32 \%$ of the websites support a second language version (i.e. English). Bearing in mind that Greece is considered to be a frequent travel destination for many tourists around the world, this is considered to be a significant limitation for Greek hospital web sites.

\section{CONCLUSIONS}

This research presents a conceptual framework to evaluate the overall performance of Hospitals websites focusing on the following aspects, information services, communication services and transaction services. The proposed framework was empirically tested in Greek Public Hospital websites. Findings revealed that less than half of the Greek Public Hospitals have invested in establishing a virtual presence. Moreover, the vast majority of current hospitals' web sites are mainly informative but lacking significant information to enable decision making by Greek health consumers on choosing a health provider. With respect to communication services, it is evident from the results that direct and interpersonal contact by traditional media (i.e. telephone, fax) is facilitated, while interactive and real-time communication with physicians or administration employees through internet based technologies is scarcely incorpotered. Moreover, not one hospital website supports transactions services to enable online routine and other activities fullfillement in a web-based virtual environment. The European Union in the "Green Paper on Public Sector Information in the Information Society" has defined e-services concept for public governmental websites in order to provide a set of standards and classifications to improve information, communication and transaction services by governmental and public websites (European Commission, 1998). Also, a "Certification Framework for Public Administration, Sites and Portals" was developed by the governmental organisation of "Information Society" on behalf of Greek Ministry of Interior in order to specify a set of directions and standards to support effective egovernment portals design and development but mainly to improve e-governmental services. In Greece, an "e-Health Consumer Trends survey" undertaken by the Institute of Computer Science in 2005 , found that $54.2 \%$ of the Internet users go online to search for information about Health and Illnesses (H\&I) (Chronaki, et al. 2006, 2007). Moreover, $59 \%$ for H\&I make their decision whether to consult a health professional partly based on information found on the Internet. Bearing in mind, that Greek public hospitals remain the main governmental authority to provide citizens with health and medical services, it is vital that 
improvements on information quality, interaction and communication of their websites are necessary in order to provide a viable and useful online environment.

\section{REFERENCES}

Ancarani, A., 2005. Towards quality e-service in the public sector: The evolution of web sites in the local public service sector. Managing Service Quality, 15 (1), 6-23

Bodkin, C. \& Miaoulis, G., 2007. EHealth information quality and ethics issues: an exploratory study of consumer perceptions. International Journal of Pharmaceutical and Healthcare Marketing, 1 (1), 2742.

Boisvert, H. \& Canon, M., 2006. Benchmarking web site functions. Benchmarking: An International Journal, $13(1 / 2), 174-189$

Chronaki, C.E., A. Kouroubali, A., Stathopoulou, A., Roumeliotaki, Orphanoudaki, E., Esterle, L., Tsiknakis, M., 2007. Internet use for health and illness in Greece. Preliminary results of a European study on eHealth consumer trends. Archives of Hellenic Medicine, 24 (5), 440-457

Cline, R.J.W. \& Haynes, K.M., 2001.Consumer health information seeking on the Internet: the state of the art", Health Educ.Res.16 (6), 671-692

European Commission, 1998. Public Sector Information: A Key Resource for Europe. Green Paper on Public Sector Information in the Information Society, COM (98) 585

Gallant, L., Irizarry, C. \& Kreps, G.L., 2007. User-Centric Hospital Web Sites: A Case for Trust and Personalization. e-Service Journal, 5 (2), 5-26

Gruca, T. \& Wakefield, D., 2004. Hospital web sites : Promise and progress. Journal of Business Research, 57 (9), 1021-1025

Hesse, B.W., Nelson, D.E., Kreps, G.L., Croyle, R.T., Arora, N.K., Rimer, B.K. \& Viswanath, K. 2005, "Trust and Sources of Health Information: The Impact of the Internet and Its Implications for Health Care Providers: Findings From the First Health Information National Trends Survey", Arch Intern Med, 165 (22), pp. 2618-2624.

Huntington, P., Nicholas, D., Gunter, B., Russell, C., Withey, R., \& Polydoratou, P., 2004. Consumer trust in health information on the web. Aslib Proceedings, 56 (6), 373-382

Jadad AR, \& Delamothe T., 2003. From electronic gadgets to better health: where is the knowledge? BMJ 2003;327: 300-1

Keating, B., Rugimbana, R. \& Quazi, A., 2003. Differentiating between service quality and relationship quality in cyberspace. Managing Service Quality, 13 (3), 217-232

Lewers, C., 2003. Online Health: Hospitals connecting with Patients on the Cyberspace. Indiana Business Magazine. Issue August 2003.

Liddell, J.,2005. Cancer-related web sites: a selective annotated guide. Reference Services Review, 33 (1), 123-134

Llinas, G., Rodriguez-Inesta, D., Mira, J. Lorenzo, S., Aibar, C. 2008. A comparison of websites from Spanish, American, and British hospitals, Methods of Information in Medicine, 47 (2), 124-130.

Lin, B., Umoh, D., 2002. E-healthcare: a vehicle of change. American Business Review, 20 (2), 27-32.

Mukherjee, A., McGinnis, J. 2007. E-healthcare: an analysis of key themes in research. International Journal of Pharmaceutical and Healthcare Marketing, 1(4), 349-363

Norum, J. 2001, "Evaluation of Norwegian cancer hospitals' Web sites and explorative survey among cancer patients on their use of the Internet", J Med Internet Res, 3 (4), e30.

Randeree, E. \& Rao, H.R., 2004. E-Health and Assurance: Curing Hospital Web Sites, International Journal of Electronic Healthcare, 1 (1), 33-46

Zeithaml,V., Parasuraman, A, \& Malhotra, A., 2002. Service quality delivery through web sites: A critical review of extant knowledge. Journal of Academy Marketing Science, 30 (4), 362-375

Zingmond, D.S., Lim, Y.W., Ettner, S.L., \& Carlisle, D.M. 2001. Information superhighway or billboards by the roadside? An analysis of hospital web sites. Western Journal of Medicine, 175(6), 385-391 